\title{
The new frontier of hepatitis C virus (HCV)-mismatched heart and lung transplantation
}

\author{
Piergiorgio Solli ${ }^{1}$, Giampiero Dolci ${ }^{1}$, V. Marco Ranieri ${ }^{2}$ \\ ${ }^{1}$ Department of Cardio-Thoracic Surgery, Policlinico S.Orsola Malpighi University Hospital, Bologna, Italy; ${ }^{2}$ Anesthesia \& Intensive Care Medicine, \\ Alma Mater Studiorum University of Bologna, Policlinico di S.Orsola, Bologna, Italy \\ Correspondence to: Dr. Piergiorgio Solli, MD, PhD. Department of Cardio-Thoracic Surgery, Policlinico S.Orsola Malpighi University Hospital, Via \\ Giuseppe Massarenti, 9 zipcode 40138 Bologna, Italy. Email: piergiorgio.solli@ausl.bologna.it; piergiorgio.solli@gmail.com. \\ Provenance: This is an invited article commissioned by the Section Editor Dr. Ran Mo (Department of Burn and Plastic Surgery, Nanjing Drum \\ Tower Hospital, Nanjing, China). \\ Comment on: Woolley AE, Singh SK, Goldberg HJ, et al. Heart and Lung Transplants from HCV-Infected Donors to Uninfected Recipients. N Engl \\ J Med 2019;380:1606-17.
}

Submitted Oct 22, 2019. Accepted for publication Nov 20, 2019.

doi: $10.21037 /$ atm.2019.11.123

View this article at: http://dx.doi.org/10.21037/atm.2019.11.123

Heart and lung transplantation remain the main treatments for patients with end-stage cardiac and respiratory failure.

Nevertheless, the major challenge in heart and lung transplantation continue to be the shortage of suitable donors that largely exceeds the available supply. As a result of such a disproportion, many potential recipients die each year whilst in the waiting list before transplantation or alternatively became too sick to undergo transplantation.

In the case of lung transplantation these data are exacerbated both by the particularly low rate $(15-20 \%)$ of suitable lungs amongst an overall low number of multiorgan brain death donors $(1,2)$ and by a wait-list mortality as high as $30-40 \%$ (3).

Considerable efforts have been made in this direction and significant advancements have been achieved with the ultimate goal of increasing organ donation and utilization utilizing new strategies.

First, the donors pool and the utilization of organs have been expanded to organs were previously considered not suitable ("marginal donors") (4). Second, potential donors have been managed by trained healthcare experts instead of unqualified professionals with particular attention to increase the number of acquired consents from families (1). Third, clinical management of brain-dead donors in the intensive unit care have been optimized adopting clinical strategies previously shown to be effective in critically ill patients (5). Fourth, hearts and lungs from donation after controlled (cDCD) or uncontrolled cardiac death (uDCD) (6) and the lobar living lung donor program (7) have been recently proposed. Fifth, ex vivo lung perfusion (normothermic ex vivo lung perfusion: EVLP) has been proposed to increase the number of available lungs through two major routes: first, the "reconditioning" of injured lungs toward clinical acceptability and second a more accurate and prolonged evaluation of marginal lungs prior to their implant; the use of an alternate list for cardiac transplantation (where two recipients lists are arranged and non-standard donor hearts, that would otherwise not been utilized, are matched with excluded potential recipients (8).

Among the more modern and innovative strategies it should be certainly included the successful use of organs obtained from donors with hepatitis $\mathrm{C}$ virus (HCV) infection in recipient candidates who do not have $\mathrm{HCV}$ infection (so-called $\mathrm{HCV}$-mismatched transplantation) as recently published by Woolley and Coll (9), Galasso and Coll (10) and Rhondalyn and Coll (11).

The concept is not new since case reports have been previously published for kidney $(12,13)$, lung $(14,15)$ and heart transplants (16,17). In 2017 the Consensus Guidelines by the American Society of Transplantation have provided recommendations in an attempt to discipline this area and better empower further research (18).

$\mathrm{HCV}$-mismatched transplantation is a fascinating clinical model for a number of reasons. First, the new generation 
of $\mathrm{HCV}$-treatment drugs provide a cure rate (defined as sustained virologic response at 12 weeks) that approach $100 \%$. Available agents such as direct-acting antiviral agents (DAA) are active against multiple $\mathrm{HCV}$ genotypes, offer a safe pharmacological and pharmacokinetic profile and present a relatively limited amount of side effects (especially on kidney and liver function) and, more importantly, have no considerable drug interactions making clinicians far away from the pioneering era of the Interferon-based treatments when endothelial or vascular injury or severe liver toxicity were common, together with the risks of exacerbating allograft rejection due to the up-regulations of the immunologic response in the recipients (16).

Second the usual pool of HCV-positive donors-due to the unprecedented public health crisis related to opioid misuse/dependence worldwide (19)—usually consists of young drug-abusers, healthier than standard donors without HCV infections and predominantly with less co-morbidity. This may theoretically reflect into an improved organ pool of donations with expected superior outcomes in terms of both graft and donor survival.

According to the WHO, in 2017, a population of 71 million of people with chronic HCV infection are globally estimated (20), hence rendering the donor pool and the number of potential donations significantly enlarged.

Although preliminary, available data should be considered encouraging especially because they markedly address a broad pool of donors.

The paper published by Woolley et al. (9) has some unique features that must be considered. First, it is a somewhat large series of patients (44 in total, 8 heart and 36 lung transplantations); second, it is one of the first report of $\mathrm{HCV}$-mismatched lung transplantations; third, the course of administration of the pan-genotypic antiviral regimen to treat recipients was started immediately after transplant via feeding nasogastric tube and it was relatively short in duration (4 weeks). With this approach the Authors achieved a surprisingly $100 \%$ viral response.

This report undoubtedly provides support for further consideration of organs from $\mathrm{HCV}$-positive donors for heart and lung transplantation candidates. However, as pointed out by the authors themselves, these results should be regarded with caution and could not be generalized for a number of reasons.

First, the follow-up time is very short (6-12 months) and nothing could be anticipated on long term outcomes.

We know from previous series that some of the recipients from $\mathrm{HCV}$ donors have an increased incidence of cardiovascular morbidity (16) as well as other non-specific metabolic complications. Moreover, the activation of the immune system related to de novo viral infection may lead to unintended consequences, including organ rejection, additional severe infections and lethal complications, especially if organ donation is extended to critically ill non selected candidates.

Second, it is not demonstrated if the short course of DAA therapy is successful in maintaining a sustained prolonged viral response or if patients are ultimately going to relapse with $\mathrm{HCV}$ infection; in case of relapse, if they can be treated again with a second course of therapy and for how long and eventually if any relapse could be avoided with a longer course regimen of cure. We also know that the risk of acute infection in the setting of immunosuppressant could occasionally lead to rapid viral replication and in order to possible benefit the patient the level of HCV RNA should be closely monitored to implement early treatment.

There are also some practical and ethical issues that could not be ignored. DDA therapy despite efficacious is expensive outside research trials; we have learnt that this treatment should be better administered in the early period after transplantation when the $\mathrm{HCV}$ viral load is small and should be accessible in advance when the suitable $\mathrm{HCV}$ donor is available. We should be also prepared to lung transplants recipients that will refuse $\mathrm{HCV}$ infected organs for several emotional and socio-ethical reasons: what happens to those who refuse? Do they remain on the waiting list as and do continue to wait for a suitably matched organ or do they drop off from the list?

All these are open questions that need to be answered in the near future as large-scale multi-centre trials assessing the utility of to accepting HCV-mismatched donors is are definitely needed.

These findings should be expanded also to people with HIV infection that can be cured thanks to the availability of triple-combination antiretroviral therapy (cARTs) (21). Most organs from HIV-positive deceased donors have been implanted into HIV-positive adult recipients, including the paediatric HIV-positive population. The large number of HIV-infected individuals on cART is an unutilised source of otherwise eligible living organ donors (21). More recent advances in this field suggest the possibility to include as potential as living organ donors HIV-infected individuals and the possibility of offering organs to HIVuninfected individuals from HIV-infected donors who are well-controlled on combination cART. While the posttransplant administration of DDAs to treat in vivo $\mathrm{HCV}$ - 
mismatched patient is fascinating, recent data propose an even more attractive alternative: the inactivation of the virus in the harvested lungs through the EVLP circuit (10). The chance to eradicate the infection ex vivo within the organ "just before" transplantation looks really smart because can virtually avoid both the costs and the toxicity or at least the drug interactions related to antiviral medication and might resolve also the psychological barrier of the recipients to accept an infected organ with the plan of cleaning it in the immediate post-transplantation recovery time.

The efficacy of EVLP in diminishing HCV levels in donor organs was already reported as a case report (14). However, since viral replication seems not to occur within the harvested graft itself, the Authors added to EVLP, a combination of light irradiation (ultraviolet $\mathrm{C}$ ) and photodynamic therapy (PDT) (methylene blue activated with red light irradiation), for pre-donation sterilization of blood components in the blood bank (22). With this technique the HCV infection was completely eradicated during the short period of time of the normothermic EVLP perfusion. This innovative technique proved to be effective both in human lungs and in pre-clinical animal lung transplant model in pigs.

Recent data suggest that ex vivo treatment to prevent donor-recipient transmission are feasible and effective in reducing post-transplant bacterial (23) and HCV load (24). However, (I) the lack of data on HIV+ donors, (II) the lack of information on intermediate and long-term outcome, leaves cARTs treatments the only option for HIV-infected tissues.

In conclusion, as organ shortages persist, the option of utilising living or deceased donor organs from people with chronic HCV or HIV + viral infections deserves attention is going to be potentially relevant since the figures are selfexplaining: currently only $15 \%$ of lungs from available donors are used (3); HCV affects $2 \%$ of North Americans and underuse of these organs is particularly relevant because if $\mathrm{HCV}$-positive donors could be safely added to the donor pool it is estimated that at least 1,000 new donors for lung transplantation would be available every year in North America alone (1). These data confirm the urgency of provide solid evidence to allow reconditioning from infection of harvested human lungs or the treatment of recipients become infected after a mismatch transplantation.

\section{Acknowledgments}

None.

\section{Footnote}

Conflicts of Interest: The authors have no conflicts of interest to declare.

Ethical Statement: The authors are accountable for all aspects of the work in ensuring that questions related to the accuracy or integrity of any part of the work are appropriately investigated and resolved.

\section{References}

1. Reeb J, Keshavjee S and Cypel M. Expanding the lung donor pool: advancements and emerging pathways. Curr Opin Organ Transplant 2015;20:498-505.

2. Punch JD, Hayes DH, LaPorte FB, et al. Organ donation and utilization in the United States, 1996-2005. Am J Transplant 2007;7:1327-38.

3. Valapour M, Paulson K, Smith JM, et al. OPTN/ SRTR 2011 annual data report: lung. Am J Transplant 2013;13:149-77.

4. Tian D, Wang Y, Shiiya H, et al. Outcomes of marginal donors for lung transplantation after ex vivo lung perfusion: A systematic review and meta-analysis. J Thorac Cardiovasc Surg 2019. [Epub ahead of print].

5. Mascia L, Pasero D, Slutsky AS et al. Effect of a Lung Protective Strategy for Organ Donors on Eligibility and Availability of Lungs for Transplantation. A Randomized Controlled Trial. JAMA 2010;304:2620-7.

6. Farr M, Truby LK, Lindower J, et al. Potential for donation after circulatory death heart transplantation in the United States: Retrospective analysis of a limited UNOS dataset. Am J Transplant 2019. [Epub ahead of print].

7. Date H. Living-related lung transplantation. J Thorac Dis 2017;9:3362-71.

8. Felker GM, Milano CA, Yager JE et al. Outcomes with an alternate list strategy for heart transplantation. J Heart Lung Transplant 2005;24:1781-6.

9. Woolley AE, Singh SK, Goldberg HJ, et al. Heart and lung transplants from $\mathrm{HCV}$-infected donors to uninfected recipients. N Engl J Med 2019;380:1606-17.

10. Galasso M, Feld JJ, Watanabe Y, et al. Inactivating hepatitis $\mathrm{C}$ virus in donor lungs using light therapies during normothermic ex vivo lung perfusion. Nat Commun 2019;10:481.

11. McLean RC, Reese PP, Acker M, et al. Transplanting hepatitis $\mathrm{C}$ virus-infected hearts into uninfected recipients: 


\section{Page 4 of 4}

A single-arm trial. Am J Transplant 2019;19:2533-42.

12. Goldberg DS, Abt PL, Blumberg EA, et al. Trial of Transplantation of HCV-Infected Kidneys into Uninfected Recipients. N Engl J Med 2017;376:2394-5.

13. Reese PP, Abt PL, Blumberg EA, et al. Twelve-month outcomes after transplant of hepatitis C-infected kidneys into uninfected recipients: a single-group trial. Ann Intern Med 2018;169:273-81.

14. Khan B, Singer LG, Lilly LB, et al. Successful Lung Transplantation From Hepatitis C Positive Donor to Seronegative Recipient. Am J Transplant 2017;17:1129-31.

15. Abdelbasit A, Hirji A, Halloran K, et al. Lung transplantation from hepatitis $\mathrm{C}$ viremic donors to uninfected recipients. Am J Respir Crit Care Med 2018;197:1492-6.

16. Gasink LB, Blumberg EA, Localio AR, et al. Hepatitis C virus seropositivity in organ donors and survival in heart transplant recipients. JAMA 2006;296:1843-50.

17. Schlendorf KH, Zalawadiya S, Shah AS, et al. Early outcomes using hepatitis C-positive donors for cardiac transplantation in the era of effective direct-acting antiviral therapies. J Heart Lung Transplant 2018;37:763-9.

18. Levitsky J, Formica RN, Bloom RD, et al. The American

\section{Solli et al. HCV-mismatched heart and lung transplantation}

Society of Transplantation consensus conference on the use of hepatitis $\mathrm{C}$ viremic donors in solid organ transplantation. Am J Transplant 2017;17:2790-802.

19. Trecki J. A Perspective Regarding the Current State of the Opioid Epidemic. JAMA Netw Open 2019;2:e187104.

20. WHO Global hepatitis report 2017. Available online: http:www.who.int/hepatitis/publications/global-hepatitisreport2017/en/ (ISBN 978-92-4-156545-5 C)

21. Botha J, Fabian J, Etheredge H, et al. HIV and Solid Organ Transplantation: Where Are we Now. Curr HIV/ AIDS Rep 2019;16:404-13.

22. Floyd RA, Schneider JE Jr, Dittmer DP. Methylene blue photoinactivation of RNA viruses. Antiviral Res 2004;61:141-51.

23. Nakajima D, Cypel M, Bonato R, et al. Ex Vivo Perfusion Treatment of Infection in Human Donor Lungs. Am J Transplant 2016;16:1229-37.

24. Cypel M, Feld JJ, Galasso M, et al. Prevention of viral transmission during lung transplantation with hepatitis C-viraemic donors: an open-label, single-centre, pilot trial. Lancet Respir Med 2019. Lancet Respir Med 2019. [Epub ahead of print].
Cite this article as: Solli P, Dolci G, Ranieri VM. The new frontier of hepatitis $\mathrm{C}$ virus (HCV)-mismatched heart and lung transplantation. Ann Transl Med 2019;7(Suppl 8):S279. doi: 10.21037/atm.2019.11.123 